\title{
artigo
}

\section{O uso da educação popular em saúde como estratégia para o controle da dengue: PET-Saúde induzindo novas práticas de formação em saúde}

The use of popular health education as a strategy to control dengue: PET-Saúde inducing new health training practices El uso de la educación popular en salud como estrategia para el control del dengue: PET-Saúde induce nuevas prácticas de capacitación en salud

\begin{abstract}
RESUMO
Tratou-se de um relato de experiência vivenciado no projeto PET-Saúde cujo objeto de intervenção foi o desenvolvimento de práticas de educação popular em saúde para ações de prevenção e controle da dengue, tendo como cenário os Conselhos Locais de Saúde (CLS). O objetivo desse estudo é descrever a experiência e a sua contribuição para o processo de formação dos futuros profissionais de saúde. As atividades foram desenvolvidas durante o ano de 2014 e ao final foram visitados 19 CLS. Identificou limitação na participação dos usuários nas instâncias de controle social; a necessidade de (re) significar as práticas de educação popular em saúde; e a importância de se intensificar a articulação ensino-serviço-comunidade a partir de experiências pautadas em novas abordagens pedagógicas, como forma de possibilitar a construção da aprendizagem que possibilite o exercício crítico do trabalho em saúde.
\end{abstract}

DESCRITORES: Educação em Saúde; Dengue; Formação em Saúde.

\begin{abstract}
It was an experience report from the PET-Saúde project whose object of intervention was the development of popular health education practices for dengue prevention and control actions, with the setting of the Local Health Councils (CLS). The purpose of this study is to describe the experience and its contribution to the process of training future health professionals. The activities were developed during 2014 and in the end 19 CLS were visited. It identified a limitation in the participation of users in instances of social control; the need to (re) signify popular health education practices; and the importance of intensifying the teaching-service-community articulation based on experiences based on new pedagogical approaches, as a way to enable the construction of learning that enables the critical exercise of health work.
\end{abstract}

DESCRIPTORS: Health Education; Dengue; Health Training.

\section{RESUMEN}

Se trata de un relato experiencia del proyecto PET-Saúde, cuyo objeto de intervención fue el desarrollo de prácticas de educación popular en salud para acciones de prevención y control del dengue, teniendo como escenarios los Consejos Locales de Salud (CLS). El objetivo de este estudio es describir la experiencia y su contribución al proceso de formación de los futuros profesionales de la salud. Las actividades se desarrollaron durante 2014 y al final se visitaron 19 CLS. Se identificó una limitación en la participación de los usuarios en las instancias de control social; la necesidad de (re) significar las prácticas de educación popular en salud; y la importancia de intensificar la articulación enseñanza-servicio-comunidad a partir de experiencias basadas en nuevos enfoques pedagógicos, como una forma para permitir una construcción del aprendizaje que permita el ejercicio crítico del trabajo en salud.

DESCRIPTORES: Educación en Salud; Dengue; Capacitación en Salud.

RECEBIDO EM: 23/02/2020 APROVADO EM: 25/02/2020

\section{Elvira Caires de Lima}

Enfermeira. Mestre em Saúde Coletiva. Doutoranda em Saúde Pública pelo Instituto de Saúde Coletiva da Universidade Federal da Bahia (ISC/UFBA). Docente do Instituto Multidisciplinar em Saúde da Universidade Federa da Bahia (IMS/UFBA). https:// orcid.org/0000-0002-1332-5459 


\section{Natália Ferreira Santos}

Bióloga. Mestre em Biologia e Biotecnologia de Microrganismo. Docente do Estado da Bahia. Secretaria de Estado da Educação do estado da Bahia. https://orcid.org/0000-0003-0602-4460

\section{José Andrade Louzado}

Enfermeiro. Mestre em Saúde Coletiva. Doutorando em Epidemiologia pela Universidade Federal do Rio Grande do Sul (UFRGS). Docente do Instituto Multidisciplinar em Saúde da Universidade Federa da Bahia (IMS/UFBA). https://orcid.org/0000-0003-4446-7051

\section{Michela Macedo Lima Costa}

Enfermeira. Mestrado em Saúde Coletiva. Docente do Curso de Medicina da Faculdade de Saúde Santo Agostinho (FASA), Vitória da Conquista- BA. https://orcid.org/0000-0003-0919-0931

\section{Camila Macedo Lima Nagamine}

Bacharel em Matemática. Mestre em Estatística. Doutoranda em Epidemiologia pela Universidade Federal do Rio Grande do Sul (UFRGS). Docente da Universidade Estadual do Sudoeste da Bahia (UESB). https://orcid.org/0000-0003-4142-6940

\section{Anna Carla Bento Sabeh Cappi}

Enfermeira. Mestranda em Enfermagem pela Universidade Federal de Mato Grosso do Sul (UFMS), Campus de Três Lagoas (CPTL). https://orcid.org/0000-0002-5312-4512

\section{Edirlei Machado dos Santos}

Enfermeiro. Doutor em Enfermagem. Programa de Pós-Graduação em Enfermagem da Universidade Federal de Mato Grosso do Sul, Campus de Três Lagoas (UFMS/CPTL). Líder do Grupo de Estudo e Pesquisa Qualitativa em Enfermagem e Saúde (GEPQES) da UFMS/CPTL. https://orcid.org/0000-0002-1221-0377

\section{INTRODUÇÃO}

A educação popular em saúde foi o aporte teórico que delineou o desenvolvimento da ação que será apresentada no presente estudo a partir de ações emanadas do Programa de Educação pelo Trabalho para a Saúde (PET-Saúde). A educação popular em saúde enquanto método, tem como ponto de partida no processo pedagógico, a valorização do saber anterior do educando a partir de suas experiências de vida. Na luta pela sobrevivência e pela transformação da realidade, as pessoas adquirem um saber sobre a sua inserção na sociedade e na natureza. Tal conhecimento fragmentado e pouco elaborado é a matéria-prima da Educação Popular. Destarte, a valorização do saber e valores do sujeito favorecem a iniciativa e participação do educando no processo de construção social do conhecimento ${ }^{(1,2)}$.

O PET-Saúde foi instituído pelo Ministério da Saúde e Ministério da Educação em 2008, tendo como eixo central fortalecer a integração entre as Instituições de Ensino Superior (IES) e os serviços públicos de saúde. Propõe reorientar o processo de formação dos novos profissionais de saúde para atuarem no âmbito do Sistema Único de Saúde (SUS), de modo a atender as reais necessidades de saúde da população brasileira. Busca, ainda, estimular as práticas de educação permanente com a qualificação dos trabalhadores que estão inseridos nos distintos cenários dos serviços públicos de saúde e, concomitantemente, ofertar uma atenção à saúde aos usuários dos serviços segundo os princípios e diretrizes que devem direcionar os processos de trabalho das esquipes de saúde ${ }^{(3,4)}$.

Este trabalho trata de um relato de experiencia a partir da operacionalização de um projeto de extensão universitária realizado por meio do PET-Saúde, "Conquista contra a Dengue: Prevenção e monitoramento da Dengue", cujo objeto de intervenção foi o desenvolvimento de práticas de educação popular em saúde para ações de prevenção e controle da dengue, tendo como cenário os Conselhos Locais de Saúde (CLS).

A dengue persiste enquanto um problema de saúde pública, sendo que a infecção pelo vírus pode ser ou não assintomática. Quando sintomática, causa uma doença sistêmica e dinâmica, com quadro clínico variando desde formas com discretos sintomas até quadros graves, podendo evoluir para o óbito ${ }^{(5,6)}$. Sua elevada transmissibilidade pode estar associada, entre outros fatores, à falta de comprometimento das pessoas com as mudanças comportamentais necessárias para seu controle, questão que perpassa pelas práticas educativas em saúde ${ }^{(6)}$.

Alguns pesquisadores têm se debruçado sobre o contexto que envolve o desenvolvimento de práticas educativas como importante estratégia para a prevenção e controle da doença no país ${ }^{(7-10)}$. A educação em saúde pode ser considerada como importante instrumento no processo de desconstrução de práticas dos sujeitos, tendo como possibilidade, mudança de posturas e consequentemente o reconhecimento da necessidade de adoção de medidas de prevenção e controle da doença $a^{(11)}$.

Neste contexto, a educação popular em saúde foi tomada como elemento central para o desenvolvimento das atividades do projeto de extensão junto à comunidade, através das reuniões dos conselhos locais de saúde. A educação popular visa trabalhar pedagogicamente o homem e os grupos envolvidos com o processo de participação popular. Possibilita a construção de formas coletivas de aprendizado de forma efetiva, que permitem uma análise crítica 
da realidade com consequente reflexão sobre as práticas e melhoria das estratégias de luta e enfretamento dos problemas ${ }^{(1,12)}$.

Considerando a relevância do trabalho desenvolvido pelo PET-Saúde a partir das práticas educativas, optou-se por um referencial teórico que possibilitasse a construção social do conhecimento (alunos-professores-trabalhadores-usuários). Nesse sentido, as noções de educação popular em saúde foram associadas a pedagogia da autonomia de Freire ${ }^{(13)}$. Partindo dessa perspectiva, definiu-se como espaço de produção do conhecimento acerca das ações de controle e prevenção da dengue, os cenários dos conselhos locais de saúde do município de Vitória da Conquista, Bahia.

Os CLS foram regulamentados pela Lei n. ${ }^{\circ} 8.142 / 90$ e Resolução $n .^{\circ}$ 333/2003 do Conselho Nacional de Saúde (CNS) como forma de ampliar o processo de comunicação entre os usuários e as unidades de saúde de determinada localidade $^{(14,15)}$. Os CLS se limitam à área de abrangência de uma determinada unidade de saúde, com o objetivo de discutir e definir com a comunidade local os principais problemas de saúde, suas formas de intervenção e a participação da comunidade. São importantes estratégias que a gestão em saúde dispõe para incentivar a mobilização comunitária, de modo a ampliar o envolvimento da sociedade com os problemas locais de saúde, assim como permear o compartilhamento de saber e poder na produção da saúde ${ }^{(16)}$.

Os CLS merecem destaque por favorecerem a participação da comunidade, principalmente por estarem próximos da população, inseridos no espaço estratégico da Atenção Primária à Saúde. A utilização dos conselhos locais como espaços de produção da saúde, na perspectiva da educação popular em saúde, pode cooperar para qualificar as iniciativas locais de controle da dengue, uma vez que os espaços dos conselhos devem ser vistos como espaços plurais para legitimação de outra concepção de saúde, de promoção do diálogo e da afirmação do popular ${ }^{(17)}$.

Deste modo, os CLS foram eleitos como espaços para a produção do conhe- cimento sobre a prevenção e controle da dengue, tendo em vista que sua composição conta com a participação de usuários que exercem liderança na comunidade. Acredita-se que a sensibilização desses sujeitos, a priori considerados proativos, permitirá maior mobilização da comunidade local, de modo a ampliar a compreensão sobre o problema da dengue, possibilitando a reflexão sobre suas práticas e contribuindo para o controle da doença.

Partindo das considerações apresentadas, questiona-se: Como as práticas de educação popular em saúde podem contribuir para o controle da dengue e com o processo de formação de novos profissionais de saúde? Este relato pretende descrever a experiência das ações de educação popular em saúde nos conselhos locais de saúde para a prevenção e controle da dengue e a sua contribuição para o processo de formação dos futuros profissionais de saúde.

\section{METODOLOGIA}

Esta experiência foi desenvolvida na região do semiárido nordestino, no município de Vitória da Conquista localizado na região Sudoeste do Estado da Bahia, distante a $503 \mathrm{Km}$ de Salvador. Possui população de 338.885 habitantes, sendo o terceiro maior município do Estado(18). O município está organizado em 11 distritos, é sede da Macrorregião de Saúde do Sudoeste da Bahia, e situa-se em entroncamentos de várias rodovias, o que o torna um polo de atração para cerca de 80 municípios das regiões circunvizinhas, incluindo o Norte de Minas Gerais ${ }^{(19)}$.

Vitória da Conquista é destaque no Estado e na Região Nordeste devido aos avanços do SUS municipal, desde final da década de 90 com o processo de municipalização da saúde, e tem sido referência para realização de diversos estudos na área de Saúde Coletiva ${ }^{(20-24)}$. A gestão de saúde municipal instituiu uma coordenação de controle social com o objetivo de acompanhar, assessorar e monitorar o desempenho do conselho municipal de saúde e dos conselhos locais de saúde. Até o ano de 2014, haviam sido formalizados 39 conse- lhos locais, presentes em todas as unidades de saúde do município, constituídos por representantes dos profissionais das unidades de saúde e os usuários do serviço, respeitando a paridade das representações.

$\mathrm{O}$ projeto de pesquisa e extensão universitária PET-Saúde, tendo como linha de pesquisa "Conquista contra a Dengue: Prevenção e monitoramento da Dengue" ao reconhecer a importância da mobilização popular para o controle da dengue, definiu como locus de trabalho para as práticas de educação popular em saúde, os espaços de discussão dos CLS. O método de trabalho pautou-se nos pressupostos difundidos por Paulo Freire ${ }^{(13)}$ cujo cerne centra-se na articulação relacional que se estabelece entre o saber popular e o saber acadêmico.

Portanto, tomou-se como referencial teórico a pedagogia da autonomia de Freire ${ }^{(13)}$ que propõe a construção do processo ensino-aprendizado pautada numa relação de respeito à autonomia do ser educando, com isso, o respeito à autonomia e à dignidade de cada um é um imperativo ético. A capacidade do ser humano de aprender, decorre da sua habilidade de apreender a substantividade do objeto aprendido ${ }^{(13)}$.

\section{RELATO DA EXPERIÊNCIA}

Para execução das atividades foi necessário estabelecer previamente uma articulação intrasetorial dentro da Secretaria Municipal de Saúde. Realizou-se a articulação entre os setores de Vigilância Epidemiológica, Coordenação da Atenção Primária à Saúde e Coordenação Municipal do Controle Social para que o projeto PET-Saúde conseguisse incluir o tema "educação popular e o controle da dengue" na pauta das reuniốes dos CLS.

As atividades de educação popular em saúde foram realizadas nos meses de janeiro a dezembro de 2014, por um grupo tutorial composto por 5 discentes do Curso de Graduação em Ciências Biológicas, dois preceptores que eram profissionais $\mathrm{da}$ rede de serviços do SUS (uma enfermeira e uma bióloga) e uma tutora acadêmica, docente da Universidade Federal da Bahia (UFBA). Todas as atividades foram plane- 
jadas a partir da aproximação e articulação ensino-serviço-comunidade. Ao final do projeto foram visitados 19 CLS, sendo que 5 destes estavam localizados em zona rural, envolvendo a mobilização de 649 participantes entre conselheiros de saúde, profissionais de saúde e usuários do SUS.

Os encontros duraram aproximadamente 40 minutos, eram conduzidos pelos discentes que realizavam rodas de conversas, de modo a promover a fala dos participantes na tentativa de estimular a reflexão sobre as práticas sanitárias da comunidade. As atividades de educação popular em saúde foram realizadas por meio de exposições dialogadas sobre a dengue em que se abordou as formas de transmissão da doença; sinais e sintomas; formas de prevenção; epidemiologia da doença no município; e a importância da mobilização popular para a eliminação de possíveis criadouros da larva do mosquito. Nos encontros foram distribuídos materiais educativos como panfletos, folders, cartazes, boletins epidemiológicos e boletins informativos com orientações sobre os sintomas e formas de controle da doença. A cada encontro, era reforçado o compromisso para que os participantes repensassem suas práticas e tornassem multiplicadores do conhecimento, de modo a contribuir para o controle e eliminação de possíveis focos de criadouros de dengue na comunidade.

\section{DISCUSSÃO}

\section{A educação popular em saúde como instrumento de produção de autono- mia dos sujeitos numa perspectiva Freireana}

As tentativas de promover o controle da dengue no Brasil vem perdurando ao longo das últimas décadas. Estratégias de controle da doença foram traçadas desde o final da década de noventa com a criação de programas específicos de alcance nacional. No entanto, as ações de prevenção durante muitos anos se pautaram em ações que priorizavam as atividades de combate ao mosquito, como o uso de inseticidas ${ }^{(7)}$. Essas práticas reproduziam um modelo de prevenção de cunho sanitarista, limitada a um modelo de reprodução da história natural da doença, baseada na interrupção do ciclo reprodutivo do vetor.

A permanência da alta incidência da doença com crescimento constante evidenciou a fragilidade dos processos de educação em saúde. Desde então, mudanças no modelo de prevenção da dengue foram instituídas incorporando uma nova abordagem preventiva que envolve práticas constantes de educação em saúde, fortalecimento das ações de vigilância, atuação intersetorial e mobilização popular ${ }^{(25,26)}$.

Ampliar o foco de atuação das medidas de prevenção da dengue é uma necessidade, tendo em vista que práticas unicamente sanitaristas e ações educativas normativas e verticalizadas não atingem mudanças comportamentais da população. Operacionalizar medidas de controle da dengue exige pensar a educação em saúde numa perspectiva popular, coletiva e corresponsável que desencadeie mudanças comportamentais.

A presente experiência de extensão universitária se constituiu a partir de práticas de educação popular em saúde, a partir do referencial teórico da Pedagogia da Autonomia de Freire ${ }^{(13)}$. Nesta perspectiva, a educação popular é um instrumento capaz de produzir mudanças comportamentais individuais e coletivas, a partir da transformação da autopercepção do sujeito e das relações que ele estabelece com o seu entorno. Desse modo, aproveitou-se os espaços de discussão dos CLS e desenvolveu-se práticas de educação popular em saúde pautadas numa perspectiva de relação dialógica com a comunidade, de modo a estimular seu envolvimento com a solução de problemas coletivos, especificamente, a dengue. A educação em saúde quando estruturada na perspectiva dialógica, emancipatória e participativa promove o empoderamento do indivíduo/comunidade, o que possibilita a promoção da cidadania, a transformação social e a qualidade de vida ${ }^{(27)}$.

Nesta perspectiva, o aporte da educação popular foi empregado por ter como alicerce a educação como instrumento político para atingir a conscientização e politização dos atores envolvidos no processo educati- vo (educador e educando); sendo compreendida como uma proposta de ação cultural direcionada à busca da libertação por meio da construção de uma prática pedagógica dialógica e problematizadora ${ }^{(28)}$.

Embora os cenários dos CLS sejam compreendidos como espaço fértil para a produção de práticas educativas e propício à indução de mudanças, segundo as características de seus participantes, geralmente com perfis de liderança na comunidade, observou-se pouca participação dos membros nas reuniões. Resultado similar foi encontrado em estudo pregresso realizado por Bispo Júnior e Martins ${ }^{(16)}$ no mesmo cenário da presente experiência. Os autores evidenciaram como um dos entraves para a efetivação do controle social, o desinteresse da população, identificado a partir da ínfima participação e o discreto envolvimento comunitário.

A discreta participação e representatividade da comunidade nas instâncias de controle social evidenciada nesse e em outros estudos ${ }^{(16,29-31)}$ apontam para a necessidade de (re)significar as representações sobre participação popular e o envolvimento comunitário, bem como superar o modelo de educação em saúde pautado nos pilares da denominada educação bancária preventivista. Fortalecer práticas de educação popular em saúde numa perspectiva da pedagogia libertadora permite estimular o interesse dos usuários dos serviços pelo aprendizado, estimulando-os a se envolverem e a se corresponsabilizarem com os problemas de saúde locais.

Percebeu-se com a presente experiência que a comunidade local não se sentia responsável pela problemática da dengue, o que fazia com que a mesma delegasse a responsabilidade pelo controle da doença à secretaria de saúde e a outros setores, por exemplo, o serviço de limpeza pública. Tal situação pôde ser constatada pelos questionamentos, durante as reuniões, relativos a visita do agente de endemias e à coleta do lixo.

Reconhece-se a importância da intersetorialidade para o controle da dengue, por meio da articulação de diversos segmentos sociais como os serviços de saúde, educação, limpeza pública, comunicação entre 
outros. No entanto, essas ações são organizadas a partir dos espaços coletivos e, parte da manutenção das epidemias da dengue está relacionada ao grande contingente de criadouros infectados permanecerem dentro das residências ${ }^{(10)}$. Neste contexto, a visita frequente do agente de endemias, a coleta regular do lixo e a limpeza dos espaços públicos de forma isolada não são suficientes para reverter o perfil epidemiológico da dengue, se os cidadãos não reconhecerem sua responsabilidade no processo de controle da doença. Portanto, é imprescindível investir e insistir na realização de atividades de educação popular em saúde numa perspectiva libertadora, que incitem mudanças no comportamento individual e no envolvimento dos sujeitos com a vigilância de suas ações.

\section{A formação de novos sujeitos colabo- radores para a construção do SUS}

A formação acadêmica construída nas universidades tem se pautado em um modelo positivista biológico-centrado que se mostra insuficiente para atender a todas as necessidades de saúde da população. $\mathrm{O}$ paradigma da medicina científica e do Modelo Flexneriano contribuiu para a formação de profissionais com alta capacidade técnica, porém com dificuldades para identificar as necessidades de saúde que extrapolem o determinismo biológico e dificuldades de lidar com problemas recorrentes da comunidade ${ }^{(27,32)}$. Partindo dessa conjuntura, o PET-Saúde propõe mudanças no modelo de formação profissional em saúde. Busca desenvolver nos discentes, competências que ultrapassam o saber técnico científico, se apropriando de outras habilidades de cunho social, ético e comunicativo. Pretende contribuir com a formação de profissionais de saúde que sejam capazes de lidar com a complexidade dos problemas de saúde das comunidades.

O presente relato de experiência é relevante, uma vez que inova por envolver no grupo do PET-Saúde estudantes do Curso de Ciências Biológicas. A grande maioria dos estudos e projetos de extensão realizados por grupos do PET tem sido desenvolvida com estudantes de Cursos da Saúde como Enfermagem, Medicina, Odontologia e Nutrição, porém não inclui o Curso de Ciências Biológicas ${ }^{(33-38)}$. A escolha pela temática dengue favoreceu a inclusão dos estudantes de Ciências Biológicas devido a interseção das suas áreas de conhecimento, a entomologia. Tal inovação contribuiu com a efetivação das práticas interdisciplinares e a produção do conhecimento coletivo.

Durante o projeto foi possível transitar e extrapolar os limites disciplinares e reconstruir novos conhecimentos pautados por uma ótica que extrapola a hierarquia disciplinar. Associado a isso, conseguiu-se vivenciar a produção do conhecimento elaborada a partir da multiplicidade de saberes considerada pela subjetivação de quem ensina e aprende ${ }^{(39)}$.

Tal experiência permitiu aos discentes do Curso de Ciências Biológicas aproximarem-se da área de conhecimento da Saúde Coletiva que ainda é pouco ou nada discutida nos currículos de graduação desse curso. As Diretrizes Curriculares Nacionais (DCN), aprovadas a partir de 2001, estabelecem elementos comuns ao ensino de todas as graduações da área da saúde, para contribuir com a formação de profissionais aptos a compreenderam a produção do cuidado em saúde a partir da integralidade da assistência ${ }^{(40)}$. Assim como, a construção de projetos políticos pedagógicos que contemplem além do conhecimento técnico-científico o compromisso ético-politico ${ }^{(41)}$. No entanto, apesar do Curso de Ciências Biológicas estar inserido dentro das 14 profissões da área da saúde, os currículos do curso permanecem aquém das discussões sobre promoção da saúde, vigilância da saúde, educação em saúde e princípios e diretrizes que regem o SUS.

Durante o desenvolvimento do projeto "Conquista contra a Dengue: Prevenção e monitoramento da Dengue" os estudantes de Ciências Biológicas tiveram a oportunidade de estudar sobre os princípios e diretrizes do SUS, vigilância à saúde, educação em saúde e controle social, conteúdos não inseridos nos componentes curriculares do curso. Ademais, puderam vivenciar a rotina de trabalho nos serviços de vigilância epidemiológica e vigilância sanitária, sendo acompanhados por uma preceptora bióloga que atua na vigilância sanitária do município. Tal experiência possibilitou vivenciar a atuação do biólogo nos serviços do SUS e ampliar o olhar e interesse dos futuros profissionais por novas áreas de atuação.

As atividades de educação popular em saúde desenvolvidas nos CLS e conduzidas pelos discentes da graduação favoreceram a articulação ensino-serviço-comunidade, um dos eixos norteadores do PET-Saúde e diretriz dos programas de extensão universitária. As práticas de extensão universitária devem nortear a produção de saberes a partir do confronto com a realidade numa dinâmica dialógica, multiprofissional e socialmente comprometida. O processo formativo em saúde, desenvolvido a partir de atividades de extensão, induz à produção de novas práticas de cuidado integral, focado não apenas nas habilidades técnicas, mas na ética e responsabilidade social ${ }^{(42)}$.

Vivenciar o planejamento e a execução de atividades de educação popular em saúde permitiu ao grupo tutorial do PET desenvolver habilidades de liderança, comunicação, trabalho em equipe, empatia, escuta qualificada, agilidade frente a situações inesperadas e aproximar-se da realidade concreta e dinâmica dos serviços de saúde. $\mathrm{O}$ processo de formação em saúde não pode ser resumido ao processo de aquisição, transmissão e difusão de conhecimentos restrito ao ambiente universitário ${ }^{(41)}$. É imperativo formar profissionais cujo saber coadune com as necessidades locais de saúde. A construção do aprendizado em espaços comunitários permite a interlocução com o usuário dos serviços de saúde, o que torna o processo ensino-aprendizagem mais produtivo para o exercício crítico do trabalho em saúde.

A formação produzida a partir da experimentação e do exercício de aprender no espaço do trabalho implica num ato pedagógico que produz sentidos e significados dos conteúdos que se estuda. $\mathrm{O}$ conhecimento é produzido a partir do resgate na memória das recordações de ações vividas ${ }^{(39)}$. 
O processo de formação produzido no e pelo encontro de pessoas gera vínculos e motiva mudanças nas práticas a partir do reconhecimento de alternativas visualizadas pelo exemplo da ação do outro. $\mathrm{O}$ encontro entre professores, alunos, profissionais e usuários possibilitou a reconstrução das práticas profissionais e do processo ensino-aprendizagem, o que permite reconhecer os limites do aprendizado baseado na transmissão de conhecimento unidirecional. A aproximação entre os diversos sujeitos polariza os papéis de quem ensina e aprende e o conhecimento não é transmitido, mas construído a partir dessas relações.

A multiplicidade desses encontros provoca mudanças na compreensão individual da significação da relação do eu com o mundo. Uma vez iniciado tal processo de mudança e induzido o processo de construção do conhecimento, a partir de uma nova abordagem pedagógica, libertadora, o sujeito que existia antes da experiência se (re)constrói e se transforma em outro sujeito autônomo, crítico-reflexivo e apto a contribuir com as reais necessidades de saúde locais.

\section{CONCLUSÃO}

A experiência do projeto de extensão "Conquista contra a Dengue: Prevenção e monitoramento da Dengue" a partir de práticas de educação popular em saúde realizadas nos Conselhos Locais de Saúde evidenciou um cenário potencial que pode ser utilizado para outras práticas de promoção da saúde. No entanto, identificou-se uma limitação na participação dos usuários e na representatividade da comunidade nas instâncias de controle social. Tal situação se reveste de elementos inerentes a uma construção sócio-histórica que permeia as representaçôes que as pessoas constroem sobre os elementos que determinam e condicionam o processo saúde-doença-cuidado.

Os CLS ainda são espaços pouco utilizados que precisam ser fortalecidos e reconhecidos como cenário legítimo de discussões e manifestações populares. Os órgãos de controle social podem tornar-se parceiros importantes para o desenvolvimento de ações de relevante impacto social, como o controle da dengue. Tais espaços de participação precisam ser melhor aproveitados para a instituição de práticas de educação popular em saúde pautadas num referencial que possibilite a construção social do conhecimento, a partir do compartilhamento de saber e poder na produção da saúde. Faz-se necessário (re) significar as práticas de educação em saúde compreendendo esta como uma estratégia capaz de produzir mudanças comportamentais individuais e coletivas, a partir da reflexão das práticas e responsabilidades sociais, utilizando-se, para tanto, de uma pedagogia libertadora e dialógica.

A presente experiência também contribui com as discussões sobre o processo de formação em saúde e evidencia a importância de se intensificar a articulação ensino-serviço-comunidade a partir de experiências pautadas em novas abordagens pedagógicas. A construção da aprendizagem a partir das relações estabelecidas entre professores-alunos-usuários-profissionais e das variações dos cenários de prática possibilita o exercício crítico do trabalho em saúde. $\mathrm{O}$ ensino-aprendizagem produzido nas práticas de extensão universitária possibilita a formação de profissionais imbuídos de habilidades de cunho ético e social e, concomitantemente, aptos a lidarem com as reais e complexas necessidades de saúde.

Os resultados da presente experiência afirmam a relevância do PET-Saúde como uma importante estratégia indutora de mudanças no modelo de formação em saúde, na articulação ensino-serviço-comunidade e no fortalecimento da política de educação permanente em saúde. Aponta para a necessidade do desenvolvimento de estudos que identifiquem e analisem os elementos que corroboram para a (des)valorização das práticas educativas em saúde por parte dos usuários, uma vez que o processo ensino aprendizado se concretizará a partir do envolvimento de todos os sujeitos participantes do processo educativo.

\section{REFERÊNCIAS}

1. Vasconcelos EM. Educação Popular: de uma Prática Alternativa a uma Estratégia de Gestão Participativa das Políticas de Saúde. Physis. $2004 ; 14(1): 67-83$.

2. Souza KM, et al. Práticas pedagógicas de Educação Popular em Saúde e a formação técnica de Agentes Comunitários de Saúde no município do Rio de Janeiro, Brasil. Interface. 2014; 18(2):1513-1522.

3. Ministério da Saúde, Ministério da Educação (BR). Portaria Interministerial n. ${ }^{\circ}$ 1.802, de 26 de agosto de 2008. Institui o Programa de Educação pelo Trabalho para a Saúde - PET - SAÚDE. Diário Oficial da União, Brasilia, DF, 27 de agosto de 2008.

4. Ministério da Saúde, Ministério da Educação (BR). Portaria Interministerial n. ${ }^{\circ}$ 421, de 03 de março de 2010. Institui o Programa de Educação pelo Trabalho para a Saúde (PET-Saúde) e dá outras providências. Diário Oficial da União. 4 Mar 2010
5. Ministério da Saúde, Secretaria de Vigilância em Saúde, Departamento de Vigilância das Doenças Transmissíveis (BR). Dengue: diagnóstico e manejo clínico: adulto e criança/ Ministério da Saúde, Secretaria de Vigilância em Saúde, Departamento de Vigilância das Doenças Transmissíveis. 5. ed. Brasilia: Ministério da Saúde, 2016.

6.GuzmanMG, HarrisE. Dengue. Lancet.2015jan;385(9966):385:453.

7. Cesarino MB, et al. A difícil interface controle de vetores - atenção básica: inserção dos agentes de controle de vetores da dengue junto às equipes de saúde das unidades básicas no município de São José do Rio Preto, SP. Saúde e sociedade. 2014; 23(3):1018-1032.

8. Girão RV, et al. Health education about dengue: contributions to the development of competencies. Revista de Pesquisa: Cuidado é Fundamental Online. 2014; 6(1):38-46.

9. Rangel-S ML Dengue: educação, comunicação e mobilização na perspec- 


\section{REFERÊNCIAS}

tiva do controle-propostas inovadoras. Interface. 2008; 12(25):433-441.

10. Sales FMS. Ações de educação em saúde para prevenção e controle da dengue: um estudo em Icaraí, Caucaia, Ceará. Ciências saúde coletiva. 2008; 13(1):175-184.

11. Gonçalves RP, et al. Contribuições recentes sobre conhecimentos, atitudes e práticas da população brasileira acerca da dengue. Saúde e sociedade. 2015; 24(2):578-593.

12. Pinheiro BC, Bittar CML. Práticas de educação popular em saúde na atenção primária: uma revisão integrativa. Cinergis. 2016; 18(1):77-82.

13. Freire P. Pedagogia da autonomia: saberes necessários à prática educativa. São Paulo: Paz e Terra; 1996. 146 p.

14. Brasil. Lei Federal n. ${ }^{\circ} 8.142$, de 28 de dezembro de 1990. Dispõe sobre a participação da comunidade na gestão do Sistema Único de Saúde - SUS e sobre as transferências intergovernamentais de recursos financeiros na área da saúde e dá outras providências. Diário Oficial da União, Brasilia, DF, 31 de dezembro de 1990.

15. Ministério da Saúde, Conselho Nacional de Saúde (BR). Resolução n. ${ }^{\circ}$ 333, de 04 de novembro de 2003. Brasilia, DF: Ministério da Saúde, 2013.

16. Bispo Junior JP, Martins PC. Envolvimento comunitário na Estratégia de Saúde da Família: dilemas entre institucionalização e efetiva participação. Physis. 2012; 22(4):1313-1332.

17. Cruz PJSC, et al. Desafios para a Participação Popular em Saúde: reflexões a partir da educação popular na construção de conselho local de saúde em comunidades de João Pessoa, PB. Saúde Soc. 2012; 21(4).

18. Instituto Brasileiro de Geografia e Estatística. Cidades. Bahia: Vitória da Conquista, 2018.

19. Vitória da Conquista. Plano Municipal de Saúde 2014-2017. Vitória da Conquista: Secretaria Municipal de Saúde, 2014.

20. Vilasbôas ALQ, Paim JS. Práticas de planejamento e implementação de políticas no âmbito municipal. Cad. Saúde Pública. 2008; 24:1239-50.

21. Cunha ABO, Vieira-da-Silva LM. Acessibilidade aos serviços de saúde em um município do Estado da Bahia, Brasil, em gestão plena do sistema. Cad. Saúde Pública. 2010; 26:725-37.

22. Santos AM, et al. Linhas do cuidado e responsabilização no PSF de um município da região Sudoeste da Bahia: um olhar analisador. In: Assis MMA, Nascimento MAA, Franco TB, Jorge MSB (Orgs.). Produção do cuidado no programa Saúde da Família: olhares analisadores em diferentes cenários. Salvador: Edufba; 2010.

23. Santos AM. Gestão do cuidado na microrregião de saúde de Vitória da Conquista (Bahia): desafios para constituição de rede regionalizada com cuidados coordenados pela Atenção Primária à Saúde. 334 f. Tese (Doutorado Saúde Pública) - Escola Nacional de Saúde Pública, Fiocruz, Rio de Janeiro, 2013.

24. Porto RTS, Bispo Júnior JP, Lima EC. Violência doméstica e sexual no âmbito da Estratégia de Saúde da Família: atuação profissional e barreiras para o enfrentamento. Physis. 2014; 24(3):787-807.

25. Ferreira BJ, et al. Evolução histórica dos programas de prevenção e controle da dengue no Brasil. Ciências saúde coletiva. 2009; 14(3):961-972.

26. Lima EC, Vilasbôas ALQ. Implantação das ações intersetoriais de mobilização social para o controle da dengue na Bahia, Brasil. Cadernos de Saúde Pública. 2011; 27(8).

27. Oliveira SRG, Wendhausen ÁLP. (Re)significando a educação em saúde: dificuldades e possibilidades da Estratégia Saúde da Familia. Trabalho, Educação e Saúde. 2014; 12(1):129-147.

28. Araújo RS. Educação popular e práticas sociais: notas introdutórias. In: Araújo RS, Cruz PJSC. Educação popular e práticas sociais: ação, processo formativo e construção do conhecimento. João Pessoa: Editora do CCTA; 2018. 298 p.

29. Cotta RMM, et al. O controle social em cena: refletindo sobre a participação popular no contexto dos conselhos de saúde. Physis. 2011; 21(3):1121-1138.

30. Shimizu HE, et al. Representações sociais dos conselheiros municipais acerca do controle social em saúde no SUS. Ciências saúde coletiva. 2013; 18(8):2275-2284.

31. Zambon VD, Ogata MN. Controle social do Sistema Único de Saúde: o que pensam os conselheiros municipais de saúde. Revista Brasileira de Enfermagem. 2013; 66(6):921-927.

32. Campos FE, Aguiar RAT, Belisário SA. A formação superior dos profissionais de saúde. In: Giovanella L, et al. (org.). Políticas e Sistema de Saúde no Brasil. Rio de Janeiro: Fiocruz; 2008. p. 1011 - 1034.

33. Teixeira S, et al. O PET-Saúde no Centro de Saúde Cafezal: promovendo hábitos saudáveis de vida. Revista Brasileira Educação Médica. 2012; 36(1):183-186.

34. Cyrino EG, et al. Ensino e pesquisa na estratégia de saúde da família: o PET-Saúde da FMB/Unesp. Revista Brasileira de Educação Médica. 2012; 36(1):92-101.

35. Ferraz L. O PET-Saúde e sua interlocução com o Pró-Saúde a partir da pesquisa: o relato dessa experiência. Revista Brasileira de Educação Médica. 2012; 36(1):166-171.

36. Silva MAM, et al. O Pró-Saúde e o incentivo à inclusão de espaços diferenciados de aprendizagem nos cursos de odontologia no Brasil. Interface. 2012; 16(42):707-717.

37. Morais FRR, et al. A importância do PET-Saúde para a formação acadêmica do enfermeiro. Trabalho, Educação e Saúde. 2012; 10(3):541-551.

38. Albuquerque GSC, et al. Educação pelo trabalho para a formação do médico. Trabalho, Educação e Saúde. 2013; 11(2):411-430.

39. Abrahao AL, Merhy EE. Formação em saúde e micropolítica: sobre conceitos-ferramentas na prática de ensinar. Interface. 2014; 18(49): 313-324.

40. Stella RCR, Puccini RF. A formação profissional no contexto das Diretrizes Curriculares nacionais para o curso de medicina. In Puccini RF, Sampaio LO, Batista NA (Orgs). A formação médica na Unifesp: excelência e compromisso social [online]. São Paulo: Editora Unifesp; 2008.

41. Biscarde DGS, Pereira-Santos M, Silva LB. Formação em saúde, extensão universitária e Sistema Único de Saúde (SUS): conexões necessárias entre conhecimento e intervenção centradas na realidade e repercussões no processo formativo. Interface. 2014; 18(48):177-186.

42. Silva AFL, Ribeiro CDM, Silva Júnior AG. Pensando extensão universitária como campo de formação em saúde: uma experiência na Universidade Federal Fluminense, Brasil. Interface. 2013; 17(45):371-384. 